\title{
Staphylococcus aureus Infections in the Paediatric Intensive Care Unit: Illustrated Cases
}

\author{
Kam Lun Hon $\mathbb{D}^{1},{ }^{1}$ Ronald C. M. Fung $\mathbb{D}^{1},{ }^{1}$ Karen K. Y. Leung $\mathbb{D}^{1}{ }^{1}$ Alexander K.C. Leung $\left(\mathbb{D}{ }^{2}\right.$ \\ Wun Fung Hui $\mathbb{D}^{1}{ }^{1}$ and Wing Lum Cheung $\mathbb{D}^{1}$ \\ ${ }^{1}$ Paediatric Intensive Care Unit, Department of Paediatrics and Adolescent Medicine, Hong Kong Children's Hospital, \\ Hong Kong, Hong Kong \\ ${ }^{2}$ Department of Pediatrics, The University of Calgary and The Alberta Children's Hospital, Calgary, Alberta T2M 0H5, Canada
}

Correspondence should be addressed to Kam Lun Hon; ehon@hotmail.com

Received 24 December 2020; Revised 18 April 2021; Accepted 16 May 2021; Published 4 June 2021

Academic Editor: Nina L. Shapiro

Copyright $(92021$ Kam Lun Hon et al. This is an open access article distributed under the Creative Commons Attribution License, which permits unrestricted use, distribution, and reproduction in any medium, provided the original work is properly cited.

Staphylococcus aureus is known to be one of the most common gram-positive microorganisms and an important pathogen associated with sepsis and toxic shock. We present four anonymized consecutive cases in a paediatric intensive care unit (PICU) to illustrate the different clinical manifestations of staphylococcal infections, including local infection versus systemic infection, toxic shock versus septic shock, and osteomyelitis. Eczema, short gut syndrome, and scald injury may be associated. Haematologic and coagulopathic abnormalities may be present. Prompt diagnosis and use of appropriate antimicrobial treatments is essential to reducing mortality and morbidity associated with staphylococcal infections.

\section{Introduction}

Staphylococcus aureus is known to be one of the most common gram-positive microorganisms and an important pathogen associated with sepsis and toxic shock in paediatric patients requiring intensive care [1]. There are different strains of Staphylococcus aureus which can cause a wide range of infections due to its extensive virulence factors [2]. We present four consecutive cases to illustrate the different clinical manifestations of staphylococcal infections, including local infection versus systemic infection, toxic shock versus septic shock, and osteomyelitis [2]. Prompt diagnosis and use of appropriate antimicrobial treatments is essential to reducing mortality and morbidity associated with staphylococcus infections.

\section{Case Series}

2.1. Case 1: An Infant with Eczema, Methicillin-Sensitive Staphylococcus aureus, and Transient Erythroblastopenia of Childhood. A 6-month-old boy with eczema presented with high fever $\left(40^{\circ} \mathrm{C}\right)$, tachycardia (heart rate 200 per minute), oliguria, and respiratory failure. He was treated with cefotaxime for presumed septic shock. Echocardiography showed mildly impaired left ventricular function. He was also noted to have hemoglobin $7.7 \mathrm{~g} / \mathrm{dL}$, absolute neutrophil $0.37 \times 10^{9} / \mathrm{L}$, and platelet $117,000 /$ $\mathrm{mm}^{3}$. He was suspicious of bone marrow failure, possibly consistent with transient erythroblastopenia of childhood (TEC) or hemophagocytic lymphohistiocytosis (HLH) or malignancy since he had gross hepatosplenomegaly and high ferritin $(1283 \mathrm{pmol} / \mathrm{L})$, though normal fibrinogen and triglyceride. An urgent bone marrow examination revealed pure red-cell aplasia with granulocytic maturation arrest and reactive changes but no malignant infiltration. The patient was stabilized with mechanical ventilation, red-cell transfusion, antibiotics, and low-dose adrenaline infusion. He was also noted to have an ulcerated lesion over his left forearm. The family had two cats, and they lived in a village house. Bed bugs were also found to be present in old furniture. Investigations did not show evidence of scrub typhus, dengue 
fever, or bartonella; but throat swab and left forearm wound swab revealed heavy growth of methicillin-sensitive Staphylococcus aureus (MSSA) and scant growth of Staphylococcus epidermidis; the minimum inhibitory concentrations are $0.25 \mu \mathrm{g} / \mathrm{ml}$ and $\leq 0.12 \mu \mathrm{g} / \mathrm{ml}$, respectively. The child's condition gradually improved after 4 days of treatment. He was able to be extubated and weaned off inotropic support. Haemoglobin, neutrophil, and platelet counts normalized over a week.

\subsection{Case 2: An Infant with Methicillin-Sensitive Staphylo-} coccus aureus Osteomyelitis. A 6-month-old infant girl, with a history of eczema and short-gut syndrome due to neonatal volvulus, requiring parenteral nutrition, was admitted to a PICU for septic shock and right ankle cellulitis. She had fever (temperature of $38.1^{\circ} \mathrm{C}$ ), tachycardia (heart beat 190 per minute), and a red swollen right ankle. Blood culture from her central venous catheter yielded methicillin-sensitive Staphylococcus aureus (MSSA). Magnetic resonance imaging (MRI) of her right ankle showed right distal tibial osteomyelitis, extending into the epiphyseal plate with a large subperiosteal collection around the distal tibia (Figure 1). The patient was treated with vancomycin and clindamycin. Urgent right tibial debridement was performed. A 6-week course of cloxacillin was added.

Pus was drained from the subperisosteal space, and cultures grew MSSA. She remained hemodynamically stable, with normal hematologic, renal, and liver functions. After the antibiotic therapy and the debridement, she returned to baseline functioning and was discharged from the PICU 3 days later to the surgical team to complete a 6-week course of antibiotics.

\subsection{Case 3: Scald Injury Complicated by Staphylococcal Toxic} Shock Syndrome. A previously healthy 21-month-old girl presented with fever and shock shortly after wound debridement for her scald burn, which was sustained to the right neck, right shoulder, and scapular region down to the right arm. The burn, sustained after accidentally pouring a boiling congee over herself, was of partial thickness (second degree) and covered $5 \%$ body surface area. She was initially admitted to the surgical ward, where she had the blisters deroofed and the wounds dressed in sterile saline gauzes. Wound debridement was scheduled to be performed in the operation theatre 3 days later. However, she developed a fever of up to $38.8^{\circ} \mathrm{C}$ just before the debridement and the temperature remained elevated $\left(38.7^{\circ} \mathrm{C}\right)$ the night after the operation. She had marked tachycardia (150-190/min) and hypotension $(89 / 40 \mathrm{~mm} \mathrm{Hg})$, and capillary refill was 5 seconds. She was lethargic and required $1 \mathrm{~L} / \mathrm{min}$ nasal oxygen to maintain the saturation. She was noted to have diffuse erythroderma over her trunk. She was suspected to have toxic shock syndrome and was transferred to the PICU for immediate intervention, including fluid resuscitation and broad-spectrum antibiotics, cefotaxime, and vancomycin. She required inotropic support with dopamine, adrenaline, and noradrenaline. The vasoactive inotropic score was up to 35. Echocardiography revealed mildly

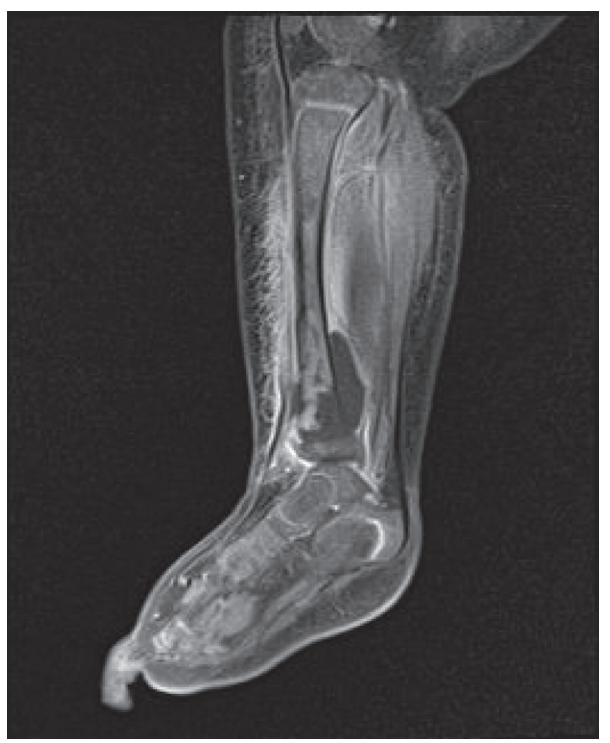

FIGURE 1: Osteomyelitis changes in the right tibial region by MRI.

impaired left ventricular function. She was intubated for suspected acute respiratory distress syndrome (lowest $\mathrm{PaO}_{2} /$ $\mathrm{FiO}_{2} 61.5, \mathrm{FiO}_{2}$ 1.0). Intravenous immunoglobulin $(1 \mathrm{~g} / \mathrm{kg} /$ day) was given for two consecutive days. Multisystem involvement was evident from elevated serum creatinine kinase $5019 \mathrm{IU} / \mathrm{L}$, raised serum alanine aminotransferase $65 \mathrm{IU} / \mathrm{L}$, lowplatelet count $89,000 / \mathrm{mm}^{3}$ (hematologic), and alterations in consciousness (central nervous system). Capillary refill time over the right sole and left chest rash was 10 seconds and 2 seconds, respectively. The capillary refill became 5 seconds over the chest whilst the rash evolved (Figure 2). Wound swab culture grew MSSA. Blood cultures remained negative. This girl met criteria for staphylococcal toxic shock syndrome, as per the 2011 case definition from the Centers for Disease Control and Prevention (CDC). Other blood investigations showed serum lactate $3.8 \mathrm{mmol} / \mathrm{L}$, serum procalcitonin $103.07 \mathrm{ng} / \mathrm{ml}$, C-reactive protein $246 \mathrm{mg} / \mathrm{L}$, prolonged prothrombin time $25.3 \mathrm{sec}-$ onds, and activated partial thromboplastin time 53 seconds with normal fibrinogen. The girl's condition gradually stabilized with mechanical ventilation, inotropic support, and antibiotic therapy. She was eventually extubated and weaned off all inotropes after 4 days.

\subsection{Case 4: A teenager with Toxic Shock and Methicillin-} Resistant Staphylococcus aureus Bacteriaemia. A previously healthy 12-year-old girl presented with a 3-day history of fever, menorrhagia, and a painful left submandibular swelling. She had a mixed picture of hemorrhagic shock and septic shock, as evidenced by tachycardia, hypotension, and anaemia (hemoglobin $4.1 \mathrm{~g} / \mathrm{dL}$ ). Respiratory, hepatic, and neurological functions were not affected. She immediately received fluid resuscitation, red-cell transfusion, noradrenaline infusion, and broad-spectrum antibiotic therapy including cefotaxime and vancomycin. Apart from anaemia, blood investigations revealed neutropenia (zero absolute neutrophil), lymphopenia (absolute lymphocyte $0.1 \times 10^{9} / \mathrm{L}$ ), and thrombocytopenia 


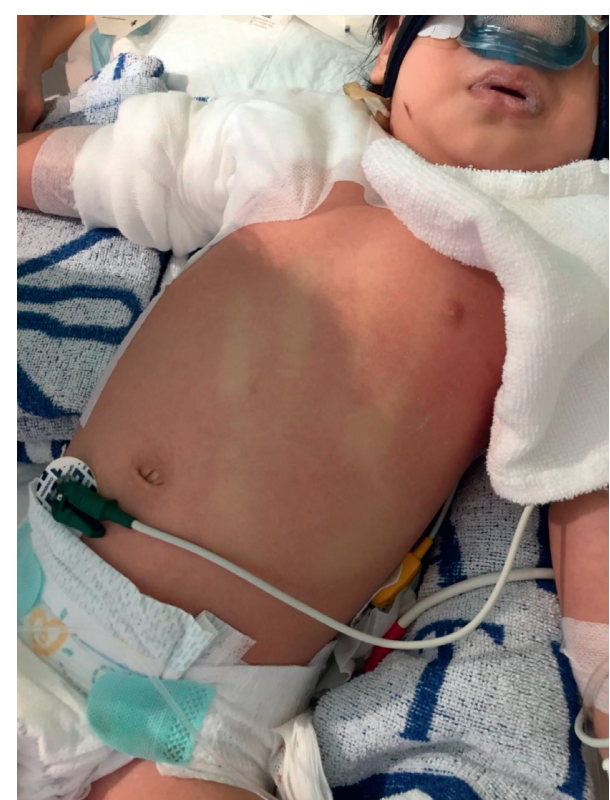

FIGURE 2: Deteriorated central capillary refill with palm mark on the torso.

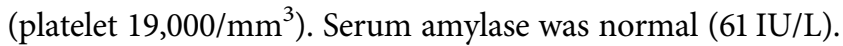
The blood culture was positive for methicillin-resistant Staphylococcus aureus (MRSA, Panton-Valentine leucocidin negative). Serologic evaluation ruled out Epstein-Barr virus, cytomegalovirus, measles, varicella-zoster virus, parvovirus, and human immunodeficiency virus. Computed tomography of the neck and thorax showed diffuse left parotid swelling with reactive inflammatory features and enlarged left upper cervical lymph nodes without abscess. Bone marrow examination revealed a markedly hypocellular marrow without hemopoietic elements, blasts, or abnormal infiltrates. The girl's condition stabilized, and she was discharged from the PICU after 4 days.

\section{Discussion}

Staphylococcus aureus is the leading cause of infection in critical-care settings accounting for significant morbidity and mortality [3]. It is capable of causing a wide range of infections in humans, from life-threatening infections in otherwise healthy individuals to nosocomial infections complicating the clinical course of patients with other primary medical or surgical disease processes $[4,5]$. There are many different strains of Staphylococcus aureus, and there is controversy over whether all strains are equally pathogenic or whether the invasive disease is associated with particular virulent genotypes [5]. The five stages in the pathogenesis of Staphylococcus aureus infections are colonization, local infection, systemic disseminated infection and/or sepsis, metastatic infection, and toxinosis. Some of these stages can be clearly identified and delineated in the cases we reviewed (Table 1) [2].

3.1. Local Infection with Systemic Effects. In the case of the infant with eczema (Case 1), the patient's presentation was atypical as he presented with septic shock, pancytopenia, and gross hepatosplenomegaly; hence, malignancy was suspected and supportive treatment for sepsis was started. Although only the wound swab was positive for Staphylococcus aureus, the septic shock was likely a result of the systemic effects from the exfoliative toxins. Transient erythroblastopenia of childhood (TEC) is an uncommon benign normocytic anaemia. Although it was observed that TEC often follows a viral-like illness, no specific aetiologic agent was identified [6]. We were unable to find any literature reporting the association of TEC with Staphylococcus aureus infections. Furthermore, TEC is uncommon in infants less than 1 year of age, and most patients with TEC are hemodynamically stable [6]. Therefore, we are of the view that TEC could be a coincidental finding in our patient with eczema infected by Staphylococcus aureus. Staphylococcus aureus is an important infectious pathogen in children with eczema, and the clinical features (especially severity and lesion intensity) are useful indicators in "predicting" moderate-to-heavy Staphylococcus aureus colonization and infection in children with eczema [7-9].

3.2. Systemic Disseminated Infection and Osteomyelitis. In Case 2, osteomyelitis was immediately suspected as the infant presented with septic shock and right ankle cellulitis. Osteomyelitis was the result of haematogenous dissemination of staphylococcal infection [10]. Early diagnosis is the cornerstone of the successful management of this disease [10]. In view of the history of eczema and need for long-term central-line use, longer duration of cloxacillin coverage is required. Empiric antibiotic treatment consists of a short intravenous cycle based on antistaphylococcal penicillin or a cephalosporin in children aged over 3 months, with the addition of gentamicin in infants aged under 3 months. An oral regimen may also be an option depending on the bioavailability of the antibiotic chosen and the assessment of clinical and laboratory data [10].

3.3. Local Infection and Toxic Shock Syndrome. For Case 3, the predisposing factor for toxic shock syndrome (TSS) was the scald injury. TSS is primarily the result of a superantigenmediated cytokine storm and $M$-protein-mediated neutrophil activation, resulting in the release of mediators leading to respiratory failure, vascular leakage, and shock [11]. It is caused by the toxin-producing strains of Staphylococcus aureus and Streptococcus pyogenes [12]. Management of TSS should be aggressive. The majority of patients with TSS will require admission to an intensive care unit for supportive care, particularly in the case of multiple organ failure [12-14]. A surveillance study in the UK reported that about $8 \%$ of TSS were associated with burns; hence, physicians should be on alert that this can be a possible cause in children with burns who suddenly become unwell [12, 14]. Antibiotic treatment should cover both Staphylococcus aureus and Streptococcus pyogenes, for example, a combination of cephalosporin, penicillin, and vancomycin. The addition of clindamycin or gentamicin can also reduce toxin production and mortality $[15,16]$. Furthermore, it is also 
TABLE 1: Staphylococcus aureus disease in the PICU.

\begin{tabular}{|c|c|c|c|c|}
\hline & Case 1 & Case 2 & Case 3 & Case 4 \\
\hline Gender & Male & Female & Female & Female \\
\hline Age & 5 months & 6 months & 21 months & 12 years \\
\hline Premorbid & Eczema & $\begin{array}{c}\text { Short-gut syndrome, } \\
\text { Broviac line, and eczema }\end{array}$ & Scald injury & Healthy \\
\hline \multicolumn{5}{|l|}{ Organ system } \\
\hline Cardiovascular & Impaired LV function & Normal & $\begin{array}{l}\text { Impaired LV function. } \\
\text { Low diastolic pressure }\end{array}$ & Low diastolic pressure \\
\hline Pulmonary & $\begin{array}{l}\text { Mechanical ventilation for } \\
\text { respiratory failure }\end{array}$ & Normal & Intubated for ARDS & Normal \\
\hline Haematologic & $\begin{array}{c}\text { Pancytopenia with gross } \\
\text { hepatosplenomegaly. Normal } \\
\text { clotting }\end{array}$ & Normal & $\begin{array}{l}\text { Thrombocytopenia. } \\
\text { Prolonged PT and aPTT }\end{array}$ & $\begin{array}{c}\text { Pancytopenia. Aplastic } \\
\text { anaemia. Normal } \\
\text { clotting }\end{array}$ \\
\hline Renal & Normal & Normal & Normal & Normal \\
\hline Hepatic & Normal & Normal & Raised ALT & Normal \\
\hline CNS & Normal & Normal & Lethargic & Normal \\
\hline $\begin{array}{l}\text { Sites of } \\
\text { Staphylococcus } \\
\text { aureus }\end{array}$ & $\begin{array}{l}\text { Throat swab (MSSA) + wound } \\
\text { swab (S. epidermidis) }\end{array}$ & $\begin{array}{c}\text { Blood culture from Broviac } \\
\text { line (MSSA), subperiosteal } \\
\text { abscess (MSSA) }\end{array}$ & Wound swab (MSSA) & Blood culture (MRSA) \\
\hline Final diagnosis & $\begin{array}{l}\text { Septic shock and TEC, possibly } \\
\text { associated with bed bug bite and } \\
\text { MSSA }\end{array}$ & MSSA, osteomyelitis & $\begin{array}{c}\text { MSSA, toxic shock } \\
\text { syndrome following scald }\end{array}$ & $\begin{array}{c}\text { MRSA septicemia } \\
\text { associated with aplastic } \\
\text { anaemia }\end{array}$ \\
\hline
\end{tabular}

ARDS: acute respiratory distress syndrome. LV: left ventricular. MSSA: methicillin-sensitive Staphylococcus aureus. MRSA: methicillin-resistant Staphylococcus aureus. TEC: transient erythroblastopenia of childhood.

important to remove the foci of bacterial toxin production as the outcomes are worse in patients who do not have the source of infection removed [12,13]. Although the use of intravenous immune globulin (IVIG) therapy in children with TSS has been controversial, the use of IVIG may be considered in patients with severe staphylococcal TSS who are unresponsive to other therapeutic measures even though the optimal IVIG regimen has not been sufficiently investigated [17].

3.4. Systemic Infection with MRSA. In Case 4, the teenager had hemorrhagic and septic shock due to MRSA bacteraemia. Pancytopenia led to a blood marrow examination that revealed aplastic anaemia. Apart from MRSA, other infectious causes for aplastic anaemia were not found. Infection is a major cause of death for patients with aplastic anaemia, while infections associated with neutropenia are a major cause of morbidity and mortality in this patient population [18]. Recovery from neutropenia is directly related to survival, and therefore, aplastic anaemia patients who are severely neutropenic should ideally be nursed in isolation, while prophylactic antibiotics and antifungals should also be considered $[19,20]$.

\section{Conclusions}

Staphylococcus aureus is an important human pathogen, and paediatricians must not overlook its potential to inflict significant morbidity and mortality [21]. The four cases illustrate severe staphylococcal disease affecting children of all ages by different pathogenesis and clinical manifestations, including local infection versus systemic infection, toxic shock versus septic shock, and osteomyelitis (Table 1). Eczema, short-gut syndrome, and scald injury may be associated. Haematologic and coagulopathic abnormalities may be present. Prompt diagnosis with aggressive support treatments and antimicrobials are essential in managing and improving clinical outcomes of these critically ill children.

\section{Data Availability}

Vital signs and some blood parameters were included in this retrospective review of anonymized cases.

\section{Ethical Approval}

Ethical approval to review mortality and morbidity of PICU admissions has been granted.

\section{Conflicts of Interest}

Professor Alexander KC Leung serves on the editorial board and is one of the academic editors of Case Report in Pediatrics. This manuscript was sent out for independent peer review.

\section{References}

[1] M. Gimenes, T. P. Salci, M. C. B. Tognim, V. L. D. Siqueira, and S. M. Caparroz-Assef, "Treating Staphylococcus aureus infections in an intensive care unit at a University Hospital in Brazil," International Journal of Clinical Pharmacy, vol. 38, no. 2, pp. 228-232, 2016. 
[2] G. L. Archer, "Staphylococcus aureus: a well-armed pathogen," Clinical Infectious Diseases, vol. 26, no. 5, pp. 1179-1181, 1998.

[3] A. P. Kourtis, K. Hatfield, J. Baggs et al., "Vital signs:epidemiology and recent trends in methicillin-resistant and in methicillin-susceptiblestaphylococcus aureusbloodstream infections-United States," MMWR. Morbidity and Mortality Weekly Report, vol. 68, no. 9, pp. 214-219, 2019.

[4] G. R. Sampedro and J. B. Wardenburg, "Staphylococcus aureus in the intensive care unit: are these golden grapes ripe for a new approach?" Journal of Infectious Diseases, vol. 215, no. 1, pp. S64-S70, 2017.

[5] D. C. Melles, R. F. J. Gorkink, H. A. M. Boelens et al., "Natural population dynamics and expansion of pathogenic clones of Staphylococcus aureus," Journal of Clinical Investigation, vol. 114, no. 12, pp. 1732-1740, 2004.

[6] M. van den Akker, Y. Dror, and I. Odame, "Transient erythroblastopenia of childhood is an underdiagnosed and self-limiting disease," Acta Paediatrica, vol. 103, no. 7, pp. e288-e294, 2014.

[7] K. L. Hon, Y. C. Tsang, N. H. Pong, C. Ng, M. Ip, and T. F. F. Leung, "Clinical features and Staphylococcus aureus colonization/infection in childhood atopic dermatitis," Journal of Dermatological Treatment, vol. 27, no. 3, pp. 1-6, 2015.

[8] K. L. Hon, K. Y. Tsang, J. S. Kung, T. F. Leung, C. W. Lam, and C. K. Wong, "Clinical signs, Staphylococcus and atopic eczema-related seromarkers," Molecular Cell, vol. 22, no. 2, p. E291, 2017.

[9] K. L. Hon, Y. C. K. Tsang, N. H. Pong, T. F. Leung, and M. Ip, "Exploring Staphylococcus epidermidis in atopic eczema: friend or foe?" Clinical and Experimental Dermatology, vol. 41, no. 6, pp. 659-663, 2016.

[10] G. Autore, L. Bernardi, and S. Esposito, "Update on acute bone and joint infections in paediatrics: a narrative review on the most recent evidence-based recommendations and appropriate antinfective therapy," Antibiotics, vol. 9, no. 8, p. 486, 2020.

[11] D. E. Low, "Toxic shock syndrome," Critical Care Clinics, vol. 29, no. 3, pp. 651-675, 2013.

[12] S. Adalat, T. Dawson, S. J. Hackett, and J. E. Clark, "Toxic shock syndrome surveillance in UK children," Archives of Disease in Childhood, vol. 99, no. 12, pp. 1078-1082, 2014.

[13] J. Zimbelman, A. Palmer, and J. Todd, "Improved outcome of clindamycin compared with beta-lactam antibiotic treatment for invasive Streptococcus pyogenes infection," The Pediatric Infectious Disease Journal, vol. 18, no. 12, pp. 1096-1100, 1999.

[14] A. P. Brown, K. Khan, and S. Sinclair, "Bacterial toxicosis/ toxic shock syndrome as a contributor to morbidity in children with burn injuries," Burns, vol. 29, no. 7, pp. 733-738, 2003.

[15] P. M. Schlievert and J. A. Kelly, "Clindamycin-induced suppression of toxic-shock syndrome-associated exotoxin production," Journal of Infectious Diseases, vol. 149, no. 3, p. 471, 1984.

[16] P. Van Langevelde, J. T. Van Dissel, C. J. Meurs, J. Renz, and P. H. Groeneveld, "Combination of flucloxacillin and gentamicin inhibits toxic shock syndrome toxin 1 production by Staphylococcus aureus in both logarithmic and stationary phases of growth," Antimicrobial Agents and Chemotherapy, vol. 41, no. 8, pp. 1682-1685, 1997.

[17] American Academy of Pediatrics, "Staphylococcus aureus," in Red Book: 2018 Report of the Committee on Infectious Diseases, D. Kimberlin, M. Brady, M. Jackson, and S. Long, Eds.,
American Academy of Pediatrics (AAP), Itasca, IL, USA, 31st edition, 2018.

[18] H. A. Torres, G. P. Bodey, K. V. I. Rolston, H. M. Kantarjian, I. I. Raad, and D. P. Kontoyiannis, "Infections in patients with aplastic anemia," Cancer, vol. 98, no. 1, pp. 86-93, 2003.

[19] J. M. Valdez, P. Scheinberg, N. S. Young, and T. J. Walsh, "Infections in patients with aplastic anemia," Seminars in Hematology, vol. 46, no. 3, pp. 269-276, 2009.

[20] S. B. Killick, N. Bown, J. Cavenagh et al., "Guidelines for the diagnosis and management of adult aplastic anaemia," British Journal of Haematology, vol. 172, no. 2, pp. 187-207, 2016.

[21] F. Miles, L. Voss, E. Segedin, and B. J. Anderson, "Review of Staphylococcus aureus infections requiring admission to a paediatric intensive care unit," Archives of Disease in Childhood, vol. 90, no. 12, pp. 1274-1278, 2005. 